\title{
Association Between Computerized Reaction Time, Short Physical Performance Battery and Berg Balance Scale in the Community-Dwelling Older Adults
}

\author{
Boram Kim ${ }^{1}$, Chang Won Won ${ }^{1}$, Jin-Young Min², Sunyoung Kim, Miji Kim ${ }^{4}$ Byung-Sung Kim ${ }^{1}$, Hyunrim Choi ${ }^{1}$, \\ Joosun Hong ${ }^{1}$ \\ ${ }^{1}$ Department of Family Medicine, College of Medicine, Kyung Hee University, Seoul, ${ }^{2}$ Institute of Health and Environment, Seoul National \\ University, Seoul, ${ }^{3}$ Department of Medicine, Graduate School, Kyung Hee University, Seoul, ${ }^{4}$ College of Medicine/East-West Medical \\ Research Institute, Kyung Hee University, Seoul, Korea
}

Corresponding Author: Chang Won Won, MD, $\mathrm{PhD}$ Department of Family Medicine, Kyung Hee University Medical Center, 23 Kyungheedae-ro, Dongdaemun-gu, Seoul 02447, Korea

Tel: $+82-2-958-8697$

Fax: +82-2-958-8699

E-mail: chunwon62@naver.com

Received: August 28, 2017 Revised: September 12, 2017 Accepted: September 13, 2017
Background: Falls, in older adults, can worsen physical functioning and lead to serious complications. Thus, as a means of preventive intervention, this high-risk group should be screened. The aim of the present study was to investigate whether reaction time is clinically applicable as a fall risk assessment tool. Methods: The study participants were community-dwelling older adults aged 65 years or older, who scored 24 or more in the Korean version of the Mini-Mental State Examination, and did not have difficulty with communication and comprehension. To investigate the relationship between hand reaction time and fall risk, we used items from a recently developed computerized apparatus, which includes 2 fall risk assessment tools: the Short Physical Performance Battery (SPPB) and Berg Balance Scale (BBS). Results: Reaction time was negatively correlated with the total SPPB $(r=-0.314, p<0.001)$ and BBS scores $(r=-0.164$, $p<0.001)$; the scores were higher when reaction times were lower. Multiple linear regression analyses also showed that the total SPPB ( $\beta=-0.30 ; 95 \%$ confidence interval $[\mathrm{Cl}],-0.26$ to -0.02$)$ and BBS scores $(\beta=-0.14 ; 95 \% \mathrm{Cl},-0.26$ to -0.02$)$ significantly increased as the reaction time decreased. Conclusion: Reaction time was significantly correlated with the SPPB and BBS, confirming the possibility of its use in tools for fall risk assessment.

Key Words: Accidental falls, Reaction time, Physical activity, Prevention

\section{INTRODUCTION}

It is well known that fall frequency tends to increase with age. It is reported that approximately a third of older adults, over the age of 65 years, experience falls at least once a year. In this population, half of those who have experienced falls experience fall recurrence, and, in approximately 10\% of all patients who have falls, a fall is accompanied by a fracture or serious complications, like traumatic brain injury and severe soft tissue injury ${ }^{1,2)}$. The complications associated with falls worsen physical functioning, and increase the use of healthcare services and mortality ${ }^{3)}$. Even if no injury occurs, a fall reduces physical activity and engenders dependence and social withdrawal. Hence, the quality of life of older adults is affected by falls themselves, and then by the fear of falls ${ }^{4)}$. Therefore, it is extremely important to prevent falls in order to maintain functional independence.

In recent times, several studies have revealed that if the multifaceted nature of falls is managed, falls can be considered predictable and preventable rather than as unavoidable accidents $^{5}$. Accordingly, studies are being conducted to develop screening tests to evaluate the physical status of older adults, and predict the fall risk level as well as the associated harmful consequences ${ }^{6,7)}$. Aging of the nervous system and musculoskeletal system, which lead to a reduction in abilities such as reaction time and balance, is suggested as the main cause of falls, and fall evaluation tools are being developed in accordance with this. However, there is a lack of studies which focus on the correlation between reaction or response time and fall risk in older adults ${ }^{8-10)}$.

Reaction time is defined as the length of time taken for an actual response to initiate after an external stimulus is perceived by the sensory neurons, and, through a series of information-processing steps, result in an action ${ }^{11)}$. Reaction time increases with age, and higher reaction times are likely to lead to falls ${ }^{12)}$.

Previously conducted studies have used reaction time pri- 
marily to assess cognitive impairment, as a fall risk factor ${ }^{13)}$. To measure reaction time, an objective test method which included the use a computer was introduced in the $1980 \mathrm{~s}^{14)}$. Reaction time is mainly used to assess cognitive informationprocessing ability. It is measured by instructing participants to make an audible sound or press a button when a visual or auditory stimulus is presented, with focus on the information processing speed of the central nervous system (CNS); however, the importance of muscular activity is minimized ${ }^{13)}$.

Reaction time involves not only the CNS, but also the peripheral neuromuscular system (PNS), which also affects balance, as described above ${ }^{15)}$. Fast muscular reaction is highly critical in recovering from imbalance caused by an unexpected disturbance, and in promptly addressing a situation in which a fall could occur ${ }^{16,17)}$. Some researchers who paid attention to this fact used ways to measure muscle and joint torque to assess muscle contraction, i.e., elements of the peripheral muscles, during the reaction time ${ }^{18,19)}$. These methods are advantageous in that they allow the velocity of the reaction by the muscular system to be quantitatively measured, but their significant drawbacks are that they are invasive, time-consuming and costly.

The present study aimed to measure reaction time using a recently developed computerized apparatus. The objective was to contrast computerized reaction time with the Short Physical Performance Battery (SPPB) and Berg Balance Scale (BBS), which are well-validated markers for fall risk.

\section{MATERIALS AND METHODS}

\section{Participants}

This study was conducted at an older adults' welfare center in Seoul, between January 18 and 29, 2016. Participants were community-dwelling older adults aged 65 years or older. Initially, those who scored 24 points or higher in the Korean version of the Mini-Mental State Examination, and were thus judged capable of comprehension and communication, were eligible to participate. To determine if the "Reaction time measurement" is meaningful in the SPPB (gold standard) through the ROC curves, $\mathrm{HO}$ is set to under the curve (AUC) of 0.5 (i.e., meaningless inspection) and assuming that $\mathrm{H} 1$ is 0.6 , the required number of participants is 334 (power $90 \%$, type 1 error is 0.05; PASS 12, NCSS, LLC, Kaysville, UT, USA) ${ }^{20)}$.

In the baseline study, there were 324 eligible participants. Of them, 13 did not want to participate. Trained investigators administered a questionnaire covering variables related to the risk of falling. Fall risk factors, as estimated in numerous studies, include hearing and vision impairment, cognitive impairment, history of falls, usage of drugs, arthritis, impairment of instrumental activities of daily living (ADL), and depression, in addition to muscle weakness and imbalance related to various chronic diseases ${ }^{1,4,5)}$. Using the questionnaire, individuals were asked about their age, sex, medication history (sleeping pills, sedatives, antidepressants, blood pressure medication, and prostatic hyperplasia medication), lifestyle habits (drinking and smoking), history of falls, and disease history (hypertension, angina, hyperlipidemia, anemia, diabetes, and sleep disorders). Exercise habits were measured using the Korean version of the International Physical Activity Questionnaire (K-IPAQ), a scale validated in older Korean adults. Body mass index (BMI) was calculated by measuring the height and weight of the participants. These were considered as control variables. A total of 287 individuals were finally included in the study after the following individuals were excluded: 8 who were taking dementia medication, 13 who were wearing orthotics, and 3 in whom BMI information was absent.

The present study utilized part of the data collected in a clinical trial (No. 2015-10-502), conducted by the Department of Family Medicine, Kyung Hee University Medical Center, in collaboration with InBody Co. Ltd., Seoul, Korea. The protocol for this study was approved by the Institutional Review Board of Kyung Hee University Hospital (approval number: 2017-02-036).

\section{Equipment and Protocol}

A recently developed computerized apparatus was used to measure reaction time. Reaction time was defined as the time taken for participants, while standing, to move their hands from a semicircular button in the center of a monitor to a randomly lighted fan-shaped button on the monitor screen. Latent reaction time was measured as the time taken from the moment one of the 4 buttons lit up on the monitor screen, to when participants lifted their hand off the semicircular button in the center in order to press the lighted button. Thus, latent reaction time is included within the length of reaction time, and indicates premotor response time (Fig. 1).

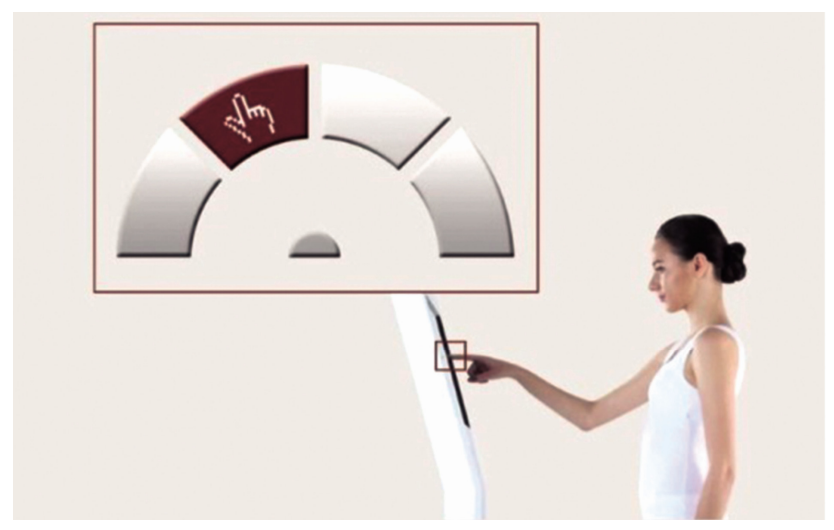

Fig. 1. Reaction time measurement. 


\section{Fall Risk Assessment}

The 2 most common fall risk assessment tools were used to evaluate balancing ability and fall risk in older adults. The SPPB is known as a battery of measures to objectively and quantitatively assess isokinetic muscular strength, and to evaluate simple physical activities ${ }^{21,22)}$. The BBS is a fall prediction tool found to be the most effective among those that evaluate balancing capability, which is the most important fall predictor ${ }^{23)}$.

\section{1) Short Physical Performance Battery}

The SPPB was used for the first time in a study conducted by the US National Institute of Aging to establish geriatric epidemiology, and is used as a method to easily evaluate physical functions. The SPPB consists of 3 items: the standing balance test, gait speed test, and repeated chair stands. On each test, a point of 0 is assigned if participants cannot perform the test, and a score between 1 and 4 is assigned, depending on the performance level, with a full score of 12 if participants succeed in all the tasks ${ }^{24)}$.

\section{(1) Standing balance test}

In the standing balance test, whether balance can be maintained for over 10 seconds is assessed with the feet placed in a side-by-side, semi-tandem, and tandem stance, respectively. If participants can hold the side-by-side or semitandem stance for more than 10 seconds, 1 point each is assigned. In the tandem stance, 1 point is assigned if a participant can hold the stance for 3-9 seconds, and 2 points if the stance is held for more than 10 seconds. The highest score is 4 .

\section{(2) Gait speed test}

Gait speed is measured by estimating how long it takes to walk $4 \mathrm{~m}$. A participant performs the test twice, and the shorter of the 2 measurements is used. If a participant cannot perform the test, a point of 0 is assigned, while the other scoring criteria are as follows: 1 point for times over 8.7 seconds, 2 points for times between 6.21 and 8.7 seconds, 3 points for times between 4.82 and 6.20 seconds, and 4 points for times under 4.82 seconds. If walking is possible using a walking aid such as a cane, the same procedure can be followed.

\section{(3) Repeated chair stands}

This test measures the time (in seconds) taken for a participant to repeatedly stand up from a chair 5 times, with the hands crossed at the chest. If the individual stands up using his/her arms, or if there are concerns about the safety of the participant, such as fall risk during the examination, the examiner can stop the examination and assign a score of
0 . The total inspection time is 60 seconds or more, and apart from a point of 0 , the other scoring criteria are as follows: 1 point for times over 16.7 seconds, 2 points for times between 13.70 and 16.69 seconds, 3 points for times between 11.20 and 13.69 seconds, and 4 points for times under 11.19 seconds.

\section{2) Berg Balance Scale}

The BBS was developed to evaluate balancing ability and fall risk in older adults. It is composed of 14 items, each scored between 0 and 4 points, with a full score of 56 points. The items are: sitting to standing, standing unsupported, sitting unsupported, standing to sitting, transfers, standing with eyes closed, standing with feet together, reaching forward with outstretched arm, retrieving object from floor, turning to look behind, turning 360 degrees, placing alternate foot on stool, standing with one foot in front, and standing on one foot. It is a useful tool to predict falls in older adults; a score in the range of 41 to 56 is classified as low-risk, a score in the range of 21 to 40 as moderate-risk, and a score in the range of 0 to 20 as high-risk ${ }^{23)}$.

\section{Statistical Analysis}

All the participants were categorized according to their general characteristics (age, sex, lifestyle habits, BMI, and disease history), and a t-test and analysis of variance were performed to test mean differences in the characteristics. To examine the associations between reaction time and latent reaction time, with the SPPB and BBS, Pearson correlation coefficients were calculated.

To further investigate the relationships, multiple linear regression analysis was performed and standardized regression coefficients (beta coefficients, $\beta$ ) and 95\% confidence intervals (95\% Cls) were computed. All the analyses were performed using SAS 9.2 (SAS Institute Inc., Cary, NC, USA), and statistical significance level was set at $\alpha=0.05$

\section{RESULTS}

The proportion of participants aged between 75 and 79 years was higher (39.4\%) than in any other age group, and the proportion of women (71.4\%) was higher. Ninety-one percent of the participants exercised a little $(<600$ metabolic equivalent $\left.\mathrm{min} / \mathrm{wk}^{25}\right)^{2}$. Those with a fall history constituted $15.3 \%$ of the participants. With respect to disease history, hypertension was the most common morbidity (57.1\%), followed by hyperlipidemia (25.4\%) and diabetes (18.1\%).

The mean total SPPB score was higher in men (10.5) than in women (9.7), and those with a sleep disorder had a significantly lower mean SPPB total score (9.3). In terms of all the other characteristics, significant differences were not obser- 
ved. The mean BBS score was significantly lower $(p<0.001)$ in patients with a hypertensive disease history $(p=0.001)$. Apart from age and hypertension, significant differences were not found (Table 1).

The Pearson correlation coefficients, computed between reaction time/latent reaction time and SPPB or BBS, showed that reaction time and latent reaction time were correlated with SPPB and BBS, and these associations were significant. Reaction time was negatively correlated with the total SPPB score $(r=-0.314, p<0.001)$ and BBS score $(r=-0.164, p<0.001)$. With regards to the constituent items of the SPPB, reaction time was positively correlated with the gait speed test

Table 1. Short Physical Performance Battery and Berg Balance Scale scores, by participants' characteristics $(n=287)$

\begin{tabular}{|c|c|c|c|c|c|}
\hline Characteristic & No. of participants & Total SPPB scores & p-value & BBS scores & p-value \\
\hline Age (yr) & & & 0.397 & & $<0.001$ \\
\hline $65-69$ & $20(7.0)$ & $10.6 \pm 1.6$ & & $54.8 \pm 1.8$ & \\
\hline $70-74$ & $77(26.8)$ & $9.8 \pm 1.7$ & & $53.7 \pm 2.7$ & \\
\hline $75-79$ & $113(39.4)$ & $10 \pm 1.7$ & & $52.8 \pm 3.3$ & \\
\hline $80-84$ & $63(22)$ & $9.8 \pm 1.7$ & & $51.8 \pm 3.7$ & \\
\hline$\geq 85$ & $14(4.9)$ & $10 \pm 1.8$ & & $51.8 \pm 2.4$ & \\
\hline Sex & & & 0.001 & & 0.147 \\
\hline Male & $82(28.6)$ & $10.5 \pm 1.6$ & & $53.3 \pm 3.0$ & \\
\hline Female & $205(71.4)$ & $9.7 \pm 1.7$ & & $52.7 \pm 3.3$ & \\
\hline Current smoking & $18(6.3)$ & $10.1 \pm 1.6$ & 0.661 & $53.5 \pm 2.5$ & 0.330 \\
\hline Current drinking & $71(24.7)$ & $10.2 \pm 1.7$ & 0.088 & $53.4 \pm 2.9$ & 0.143 \\
\hline $\mathrm{PA}<600$ (METS/wk) & $262(91.3)$ & $9.9 \pm 1.7$ & 0.097 & $52.8 \pm 3.3$ & 0.063 \\
\hline $\operatorname{BMI}\left(\mathrm{kg} / \mathrm{m}^{2}\right)$ & & & 0.306 & & 0.488 \\
\hline Underweight $(<18.5)$ & $6(2.1)$ & $9.3 \pm 2.2$ & & $52.5 \pm 3.0$ & \\
\hline Normal weight (18.5-24.9) & $135(47)$ & $9.9 \pm 1.7$ & & $53 \pm 3.1$ & \\
\hline Overweight (25.0-29.9) & $134(46.7)$ & $10 \pm 1.7$ & & $53 \pm 3.3$ & \\
\hline Obesity $(\geq 30.0)$ & $12(4.2)$ & $9.2 \pm 1.6$ & & $51.5 \pm 4.3$ & \\
\hline History of falls & & & 0.317 & & 0.664 \\
\hline Yes & $44(15.3)$ & $9.7 \pm 1.8$ & & $53 \pm 2.6$ & \\
\hline No & $243(84.7)$ & $10 \pm 1.7$ & & $52.9 \pm 3.3$ & \\
\hline \multicolumn{6}{|l|}{ History of chronic disease } \\
\hline Hypertension & $164(57.1)$ & $9.8 \pm 1.7$ & 0.135 & $52.4 \pm 3.5$ & 0.001 \\
\hline Angina & $5(1.7)$ & $9.9 \pm 2$ & 0.926 & $53.2 \pm 2.1$ & 0.756 \\
\hline Hyperlipidemia & $73(25.4)$ & $9.9 \pm 1.7$ & 0.979 & $53.1 \pm 3.0$ & 0.561 \\
\hline Anemia & $5(1.7)$ & $10 \pm 1$ & 0.937 & $52.8 \pm 2.8$ & 0.949 \\
\hline Diabetes & $52(18.1)$ & $10 \pm 1.8$ & 0.782 & $52.2 \pm 3.4$ & 0.103 \\
\hline Sleeping disorder & $23(8.0)$ & $9.3 \pm 1.6$ & 0.045 & $51.7 \pm 4.1$ & 0.074 \\
\hline
\end{tabular}

Values are presented as number of participants (\%) or mean \pm standard deviation.

SPPB, Short Physical Performance Battery; BBS, Berg Balance Scale; PA, Physical Activity; METS, metabolic equivalent of tasks; BMI, body mass index.

p-value was calculated by the t-test or analysis of variance to compare group means.

Table 2. Correlation coefficients between reaction time, and Short Physical Performance Battery and Berg Balance Scale scores

\begin{tabular}{lccccc}
\hline \hline \multirow{2}{*}{ Score } & \multicolumn{2}{c}{ Reaction time } & & & \multicolumn{2}{c}{ Latent reaction time } \\
\cline { 2 - 3 } \cline { 5 - 6 } Total SPPB scores & Coefficient & p-value & & Coefficient & p-value \\
\hline Gait speed test & -0.314 & $<0.001$ & -0.257 & $<0.001$ \\
Chair stand test & 0.315 & $<0.001$ & & 0.266 & $<0.001$ \\
Balance test & 0.316 & $<0.001$ & & 0.251 & $<0.001$ \\
BBS scores & -0.169 & 0.004 & & -0.143 & 0.013 \\
\hline
\end{tabular}

SPPB, Short Physical Performance Battery; BBS, Berg Balance Scale. 

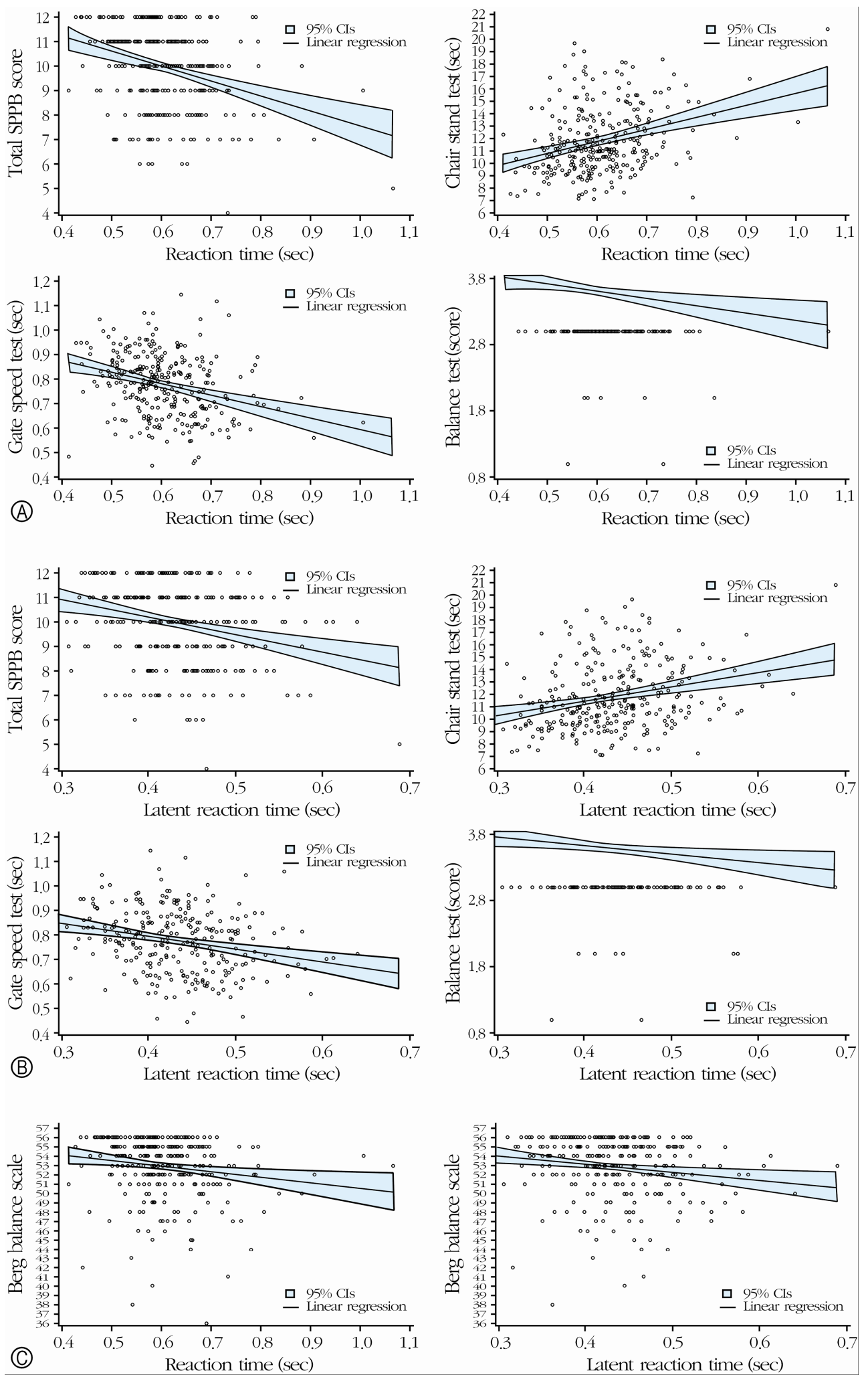

Fig. 2. Pearson correlation coefficients between reaction time and Short Physical Performance Battery (SPPB) scores (A) and the latent reaction time and SPPB scores (B). Correlation coefficients computed between reaction time/latent reaction time and Berg Balance Scale (BBS) (C). CI, confidence interval. 
Table 3. Standardized regression coefficient (95\% CIs) for reaction time and latent reaction time, by SPPB and BBS scores

\begin{tabular}{|c|c|c|c|c|c|c|c|c|}
\hline \multirow{3}{*}{ Score } & \multicolumn{4}{|c|}{ Reaction time } & \multicolumn{4}{|c|}{ Latent reaction time } \\
\hline & \multicolumn{2}{|c|}{ Unadjusted model } & \multicolumn{2}{|c|}{ Adjusted model ${ }^{*}$} & \multicolumn{2}{|c|}{ Unadjusted model } & \multicolumn{2}{|c|}{ Adjusted model* } \\
\hline & $\beta(95 \% \mathrm{CI})$ & p-value & $\beta(95 \% \mathrm{CI})$ & p-value & $\beta(95 \% \mathrm{CI})$ & p-value & $\beta(95 \% \mathrm{CI})$ & $\mathrm{p}$-value \\
\hline Total SPPB scores & $-0.31(-0.42$ to -0.20$)$ & $<0.001$ & $-0.30(-0.42$ to -0.18$)$ & $<0.001$ & $-0.26(-0.37$ to -0.15$)$ & $<0.001$ & $-0.23(-0.35$ to -0.11$)$ & $<0.001$ \\
\hline Gait speed test & $-0.31(-0.42$ to -0.20$)$ & $<0.001$ & $-0.31(-0.43$ to -0.19$)$ & $<0.001$ & $-0.25(-0.36$ to -0.14$)$ & $<0.001$ & $-0.24(-0.35$ to -0.12$)$ & $<0.001$ \\
\hline Chair stand test & $0.32(0.21$ to 0.43$)$ & $<0.001$ & $0.32(0.20$ to 0.44$)$ & $<0.001$ & $0.27(0.15$ to 0.38$)$ & $<0.001$ & $0.25(0.13$ to 0.37$)$ & $<0.001$ \\
\hline Balance test & $-0.17(-0.28$ to -0.05$)$ & 0.004 & $-0.13(-0.25$ to 0.00$)$ & 0.043 & $-0.14(-0.26$ to -0.03$)$ & 0.015 & $-0.12(-0.24$ to 0.00$)$ & 0.059 \\
\hline BBS scores & $-0.16(-0.28$ to -0.05$)$ & 0.005 & $-0.14(-0.26$ to -0.02$)$ & 0.025 & $-0.17(-0.28$ to -0.05$)$ & 0.004 & $-0.14(-0.26$ to -0.03$)$ & 0.015 \\
\hline
\end{tabular}

CI, confidence interval; $\beta$, regression coefficient; SPPB, Short Physical Performance Battery; BBS, Berg Balance Scale.

*Adjusted for age, sex, current smoking, current alcohol consumption status, physical activity, body mass index, and history of falling and chronic diseases, including hypertension, angina, hyperlipidemia, anemia, diabetes, and sleeping disorders.

$(r=0.315, p<0.001)$ and chair stand test $(r=0.316, p<0.001)$, and negatively correlated with the balance test $(r=-0.169$, $\mathrm{p}=0.004$ ) (Table 2). Fig. 2 displays the results of Table 2 in a graphical form.

Similar findings were observed even after control variables were included in the model (such as age, sex, current smoking status, current alcohol consumption status, physical activity status, BMI, as well as history of falls and chronic diseases, such as hypertension, angina, hyperlipidemia, anemia, diabetes, and sleep disorders) (Table 3).

\section{DISCUSSION}

The results of this study demonstrated that reaction time and latent reaction time showed significant relationships with SPPB and BBS. That is, as reaction time decreased, fall risk decreased, as measured by the SPPB and BBS.

Reaction time is a result of complicated actions by the CNS and PNS ${ }^{15)}$. It is determined by the sensory system, perceptual mechanism, central information processing, appropriate strategy production, information transfer with regards to appropriate reaction, and muscular action ${ }^{26,27)}$. Reaction time can be divided into premotor time (PMT) and electromechanical delay (EMD). PMT is the time from external stimulation to initial muscle activation, while EMD is the time from initial muscle activation to joint torque. In other words, PMT indicates the reaction time due to the neuronal component, and EMD indicates the reaction time due to the muscular component. Therefore, reaction time can be used to quantitatively measure the velocity of a reaction by the nervous and muscular systems involved in a fall ${ }^{28}$. Previously conducted studies have shown that measuring the response time minimizes the movement of the muscles and thus influences the $\mathrm{CNS}^{13)}$. However, the reaction time and latent reaction time measured in this study indicated PMT and EMD, respectively, and a strength of the study is that it included the influence of the PNS as well as the CNS, unlike in the case of the aforementioned study.

The information processing speed in older adults is very slow compared to younger individuals; this may be attributed to the reduced neuronal density in the brain and the distribution of neural networks due to aging, which cause difficulties in the planning and execution of autonomous movements ${ }^{27,29)}$. Other studies which investigated slow movements in cognitively impaired older adults reported that the reaction time is slow in older adults as the peripheral processing speed decreases due to the aging of the muscular system ${ }^{18,30)}$. Thus, we reason that overall reaction time is affected in a complicated fashion, through decreased central information-processing speed, and slower muscle contraction in peripheral processing, caused by muscle weakness due to aging ${ }^{15,16)}$. It is also reported that reaction time affects balance, and that a high reaction time is a risk factor for falls ${ }^{16)}$. The results of our study indicate that reaction time could be an interesting predictor of falls in older adults, due to the sensory and motor components associated with it.

In recent times, fall assessment tools based on stepping reaction time have been developed ${ }^{8-10)}$; however, those in which hand reaction time is integrated are lacking. This study distinguishes the measurement of reaction time using the hands.

However, our study has some limitations. First, due to its cross-sectional design, it was difficult to investigate any causal relationships between reaction time and falls. In addition, we attempted to analyze the average value of fall history and number of falls, but no meaningful result was obtained. Second, the study was conducted for adults over the age of 65 years, living in particular areas in Seoul, and, therefore, it is difficult to generalize the study findings to all older Korean adults. Finally, even though the results have shown that reaction time, as measured by this tool, is significantly correlated with the SPPB and BBS, validation through follow-up studies is required. We are in the process of preparing a paper focusing on the relationship between fall and reaction time, including follow-up.

In conclusion, we found that both reaction time and latent reaction time showed significant correlation with the SPPB and BBS. That is, as the reaction time decreased, the risk 
of falls decreased, as measured by the SPPB and BBS. This demonstrates the possibility of utilizing reaction time in tools for fall risk assessment.

Conflicts of Interest Disclosures: The researchers claim no conflicts of interest.

\section{Acknowledgements}

This study was funded by InBody Co., Ltd., Seoul, Korea.

\section{REFERENCES}

1. Tinetti ME. Clinical practice. Preventing falls in elderly persons. N Engl J Med 2003;348:42-9.

2. Kim KI, Jung HK, Kim CO, Kim SK, Cho HH, Kim DY, et al. Evidence-based guideline for fall prevention in Korea. J Korean Geriatr Soc 2016;20:1-28.

3. Rubenstein LZ. Falls in older people: epidemiology, risk factors and strategies for prevention. Age Ageing 2006;35 Suppl 2:ii37-41.

4. Scheffer AC, Schuurmans MJ, van Dijk N, van der Hooft T, de Rooij SE. Fear of falling: measurement strategy, prevalence, risk factors and consequences among older persons. Age Ageing 2008; 37:19-24.

5. Lajoie Y, Gallagher SP. Predicting falls within the elderly community: comparison of postural sway, reaction time, the Berg balance scale and the Activities-specific Balance Confidence (ABC) scale for comparing fallers and non-fallers. Arch Gerontol Geriatr 2004;38:11-26.

6. Abellan van Kan G, Rolland Y, Houles M, Gillette-Guyonnet S, Soto M, Vellas B. The assessment of frailty in older adults. Clin Geriatr Med 2010;26:275-86.

7. Gallagher B, Corbett E, Freeman L, Riddoch-Kennedy A, Miller S, Smith C, et al. A fall prevention program for the home environment. Home Care Provid 2001;6:157-63.

8. Pijnappels M, Delbaere K, Sturnieks DL, Lord SR. The association between choice stepping reaction time and falls in older adults: a path analysis model. Age Ageing 2010;39:99-104.

9. Ejupi A, Brodie M, Gschwind YJ, Schoene D, Lord S, Delbaere $\mathrm{K}$. Choice stepping reaction time test using exergame technology for fall risk assessment in older people. Conf Proc IEEE Eng Med Biol Soc 2014;2014:6957-60.

10. Lord SR, Fitzpatrick RC. Choice stepping reaction time: a composite measure of falls risk in older people. J Gerontol A Biol Sci Med Sci 2001;56:M627-32.

11. Stelmach GE, Amrhein PC, Goggin NL. Age differences in bimanual coordination. J Gerontol 1988;43:P18-23.

12. Gottsdanker R. Age and simple reaction time. J Gerontol 1982; 37:342-8.

13. Liu-Ambrose TY, Ashe MC, Graf P, Beattie BL, Khan KM. Increased risk of falling in older community-dwelling women with mild cognitive impairment. Phys Ther 2008;88:1482-91.
14. Zsembik BA, Peek MK. Race differences in cognitive functioning among older adults. J Gerontol B Psychol Sci Soc Sci 2001; 56:S266-74.

15. Sturnieks DL, St George R, Lord SR. Balance disorders in the elderly. Neurophysiol Clin 2008;38:467-78.

16. Lord SR, Ward JA, Williams P, Anstey KJ. Physiological factors associated with falls in older community-dwelling women. J Am Geriatr Soc 1994;42:1110-7.

17. Roberts BL. Effects of walking on reaction and movement times among elders. Percept Mot Skills 1990;71:131-40.

18. Kim JW, Jeong HY, Kim HH, Kwon YR, Eom GM, Lee JW, et al. Comparison of reaction times of ankle joint muscles in the elderly men and women. J Biomed Eng Res 2012;33:163-8

19. Larsson L, Grimby G, Karlsson J. Muscle strength and speed of movement in relation to age and muscle morphology. J Appl Physiol Respir Environ Exerc Physiol 1979;46:451-6.

20. Muir SW, Berg K, Chesworth B, Speechley M. Use of the Berg Balance Scale for predicting multiple falls in community-dwelling elderly people: a prospective study. Phys Ther 2008;88: 449-59.

21. Guralnik JM, Simonsick EM, Ferrucci L, Glynn RJ, Berkman LF, Blazer DG, et al. A short physical performance battery assessing lower extremity function: association with self-reported disability and prediction of mortality and nursing home admission. J Gerontol 1994;49:M85-94.

22. Lajoie Y, Teasdale N, Bard C, Fleury M. Attentional demands for static and dynamic equilibrium. Exp Brain Res 1993;97: 139-44.

23. Bogle Thorbahn LD, Newton RA. Use of the Berg Balance Test to predict falls in elderly persons. Phys Ther 1996;76:576-83.

24. Guralnik JM, Ferrucci L, Simonsick EM, Salive ME, Wallace RB. Lower-extremity function in persons over the age of 70 years as a predictor of subsequent disability. $\mathrm{N}$ Engl J Med 1995;332:556-61.

25. Ainsworth BE, Haskell WL, Whitt MC, Irwin ML, Swartz AM, Strath SJ, et al. Compendium of physical activities: an update of activity codes and MET intensities. Med Sci Sports Exerc 2000;32(9 Suppl):S498-504.

26. Welford AT. Causes of slowing of performance with age. In: Mackay IR, editor. Multidisciplinary gerontology: a structure for research in gerontology in a developed country. Basel: Karger; 1977. p. 43-51

27. Spirduso WW, Francis KL, MacRae PG. Physical dimensions of aging. Champaign (IL): Human Kinetics; 2005.

28. Laroche DP, Knight CA, Dickie JL, Lussier M, Roy SJ. Explosive force and fractionated reaction time in elderly low- and highactive women. Med Sci Sports Exerc 2007;39:1659-65.

29. Clarkson PM. The effect of age and activity level on simple and choice fractionated response time. Eur J Appl Physiol Occup Physiol 1978;40:17-25.

30. Welford AT, Norris AH, Shock NW. Speed and accuracy of movement and their changes with age. Acta Psychol (Amst) 1969; 30:3-15. 